\title{
Féeries
}

Études sur le conte merveilleux, XVII ${ }^{\mathrm{e}} \mathrm{XIX} \mathrm{X}^{\mathrm{e}}$ siècle

\section{Une leçon de magie économique}

Féerie, économie et politique dans l'Histoire du Prince Titi de SaintHyacinthe (1736)

A Lesson of Economic Magic: Faerie, Economy and Politics in Saint-Hyacinthe's Histoire du Prince Titi (1736)

\section{Aurélia Gaillard}

\section{(2) OpenEdition}

\section{Journals}

Édition électronique

URL : http://journals.openedition.org/feeries/708

DOI : $10.4000 /$ feeries.708

ISSN : 1957-7753

Éditeur

UGA Éditions/Université Grenoble Alpes

Édition imprimée

Date de publication : 1 juillet 2009

Pagination : 131-150

ISBN : 978-2-84310-140-3

ISSN : $1766-2842$

\section{Référence électronique}

Aurélia Gaillard, «Une leçon de magie économique », Féeries [En ligne], 6| 2009, mis en ligne le 15 septembre 2010, consulté le 07 septembre 2020. URL : http://journals.openedition.org/feeries/708 ; DOl : https://doi.org/10.4000/feeries.708

\section{(c) Féeries}




\title{
TITI ET LA FÉE ÉCONOMIE
}

Prenant en compte l'importance exceptionnelle du vaste conte-roman, l'Histoire du Prince Titi de Thémiseul de Saint-Hyacinthe, publié en I736, pour qui s'intéresse à l'articulation des nouveaux savoirs économiques et du genre du conte, nous avons constitué un "dossier " qui permet à la fois au lecteur de connaître mieux le récit (pas encore rééditér) et son auteur, grâce à l'apport dans le premier article d'éléments biographiques et d'une Annexe qui comprend le résumé de l'ouvrage, et surtout grâce à la conjonction de deux études qui présentent des points de vue complémentaires mais aussi dissonants à partir d'un même constat : l'insertion-intrication dans le conte d'un matériau économico-politique.

NDA

\author{
Aurélia Gaillard
}

Université Michel de Montaigne-Bordeaux 3

UNE LEÇON DE MAGIE ÉCONOMIQUE FÉERIE, ÉCONOMIE ET POLITIQUE DANS

L'HISTOIRE DU PRINCE TITI DE SAINT-HYACINTHE (I736)

\begin{abstract}
1 u ceevr politique de l'Histoire du Prince Titi de Thémiseul de Saint-Hyacinthe, au Livre 5 de ce long conte-roman qui en 1 dénombre huit en tout (en fait, sept de la main de l'auteur et un
court huitième par Mayer, l'éditeur du Cabinet des fées ${ }^{2}$ qui donne, selon lui, au roman " une conclusion nécessaire $\left.{ }^{3} »\right)$, conte-roman d'ailleurs tenu par la critique comme l'une des toutes premières fictions à proposer un
\end{abstract}

I. Le volume de Contes rococo, dirigé par Anne Defrance et Aurélia Gaillard, qui comprendra l'Histoire du Prince Titi, est en préparation dans la collection «La Bibliothèque des Génies et des Fées ", chez Champion.

2. Le Cabinet des Fées, ou Collection choisie des contes des fées et autres contes merveilleux, tomes 27 et 28, Amsterdam et Paris, 1786.

3. Ibid., t. 28 , p. 383 .

Féeries, $\mathrm{n}^{\circ}$ 6, 2009, p. I3I-150. 
portrait de despote éclairé ${ }^{4}$, on trouve ce bref et apparemment anodin échange entre trois personnages clefs de l'histoire, deux rois, qui sont aussi deux modèles distincts de pouvoir politique (j'y reviendrai), et une fée :

Qu'est-ce que c'est que la magnificence des plus grands rois du monde, disait Forteserre à Titi, en comparaison de la magnificence des fées ? Mais qu'est ce que c'est que cette magnificence même, dit la fée ? Qu'une vaine illusion dont les faux grands sont enorgueillis et occupés, comme les enfants de leurs jouets, car tout est relatif, et un prince qui connaît la véritable grandeur, regarde bien toutes ces choses-là comme des bagatelles's.

La réflexion signe, en effet, la mise en miroir et en perspective des pouvoirs féerique et politique dans Titi. Les pouvoirs féerique et politique sont tous deux, également, $\mathrm{du}$ "faux merveilleux ", expression que je reprends à Fontenelle dans De l'Origine des Fables ${ }^{6}$, et qui ne signifie pas un merveilleux trompeur mais un faux enchanteur. Et il ne s'agit pas seulement du pouvoir féerique (de la merveille) ou politique dans le conte mais bien sûr aussi du pouvoir féerique et politique $d u$ conte, comme l'attestent les allusions claires à Perrault (" ces bagatelles n'étaient pas de pures bagatelles ", Préface des Contes en vers de I695) et avant lui à la Fontaine (au moins dans deux fables bien connues, Le Statuaire et la statue de Jupiter et Le Pouvoir des fables ${ }^{7}$ ). Qu'est-ce à dire ? Quel enjeu cette conception a-t-elle sur le politique ou l'économique d'une part et sur le féerique d'autre part?

D'abord, cela implique que tout savoir, en l'occurrence économique et politique, est toujours tricoté dans la trame féerique ${ }^{8}$ : le pouvoir comme savoir en acte est celui du conte (de la fable), la leçon, pour être féerique,

4. Voir Jacques Barchilon, Uses of the fairy-tale in the I8th century, Genève, Studies on Voltaire, 24, 1963, p. I24 et Elisabeth Carayol, Thémiseul de Saint-Hyacinthe, Oxford, SVEC, n 221, I984, p. I49-I5O.

5. Thémiseul de Saint-Hyacinthe, Histoire du Prince Titi, dans Le Cabinet des Fées, ou Collection choisie des contes des fées et autres contes merveilleux, Amsterdam et Paris, 1786 (tomes 27 et 28), tome 28, p. 2II. Les citations se réferent toutes à cette édition.

6. Fontenelle : «Mais assurément ce sera encore bien pis quand ils [les faits] passeront de bouche en bouche ; chacun en ôtera quelque petit trait de vrai, et y en mettra quelqu'un de faux et principalement $d u$ faux Merveilleux qui est le plus agréable. " (De l'Origine des fables, dans CEuvres diverses, Paris, Brunet, 1724, t. I, p. 569, je souligne)

7. «Il était enfant en ceci/ Les enfants n'ont l'âme occupée/ Que du continuel souci/ Qu'on ne fâche point leur poupée " (Le Statuaire et la statue de Jupiter, IX, 6) et "Au moment que je fais cette moralité/ Si Peau d'âne m'était conté/ J'y prendrais un plaisir extrême/ Le monde est vieux, dit-on : je le crois, cependant/ Il le faut amuser encor comme un enfant " (Le Pouvoir des fables, VIII, 4).

8. Je renvoie au numéro 3 de Féeries consacré à la question (Politique du conte, 2006), notamment à l'article d'Anne Defrance, "La politique du conte aux XVII ${ }^{\mathrm{e}}$ et $\mathrm{XVIII}^{\mathrm{e}}$ siècles : pour une lecture oblique ", p. I3-4I. 
n'en est pas moins grave et concerne le monde - éventuellement le monde politique et économique. Cependant la "leçon " fait aussi retour sur la conception même du pouvoir économico-politique qu'elle modélise. Saint-Hyacinthe reprend mais aussi met à distance Perrault : pour qui sait distinguer la vraie grandeur de la fausse magnificence, ces « merveilles »-là, qu'elles relèvent du féerique ou du politique, sont bien des bagatelles. Le jeu de miroir entre le féerique et l'économico-politique dans le conte de Saint-Hyacinthe laisse le champ libre à une autre conception du politique, précisément débarrassé de toute féerie, c'est-à-dire exempte à la fois des manipulations et de la pseudo magie et aussi, plus simple, non aristocratique. La question reviendrait alors à déterminer l'identité de ce "prince qui connaît la véritable grandeur » : dans ou hors du conte? Titi ? Forteserre ? Ou Frederick, prince de Galles, fils et héritier du roi d'Angleterre, George II, à qui Saint-Hyacinthe dédie le Recueil de divers écrits qu'il publie la même année que l'Histoire du Prince Titi, avec une épître dédicatoire qui propose justement un portrait de despote éclairé ? Ou encore un lecteur, futur citoyen modèle, qu'il appelle de ses vœux ?

Mais, du même coup, la désignation, dans ou hors du conte, d'un modèle ou d'un support à un modèle économico-politique, implique la désagrégation même du matériau féerique : si le savoir est ailleurs, alors, le conte n'a plus raison d'être. Et c'est précisément dans cette tension et cette indécidabilité que l'Histoire du prince Titi trouve son originalité : tendu vers le roman allégorique, parfois vers l'utopie, Titi persiste dans le genre souple du conte et par là même conserve son caractère de fable expérimentale où les savoirs se questionnent en même temps qu'ils s'élaborent.

Ce qui conduit à deux dernières remarques préliminaires : le merveilleux dans Titi est d'abord/encore un "fabuleux " (au sens où l'emploie La Fontaine, sens qui s'est largement défait après la Querelle de la Fable dans les années I700-I7IO, i.e. un récit à double articulation, littérale et figurée, un merveilleux allégorique). En ce sens, ce texte en rappelle un autre, un peu plus connu à cause de la notoriété de son auteur, Arsace et Isménie de Montesquieu ${ }^{9}$, écrit sans doute dans le cadre du salon de $\mathrm{M}^{\text {elle }}$ de Charolais vers 1742, qui propose également des " maximes " de bien gouverner et un cadre fictif (oriental en l'occurrence) d'où est cette fois-ci absolument exclue toute magie, le seul élément merveilleux (les ressources financières des amants) étant finalement expliqué de manière rationnelle à la fin du conte-roman. Bien sûr, le rapprochement n'est

9. Je renvoie à mon étude " Montesquieu et le conte oriental - L'expérimentation du renversement ", dans Féeries, $\mathrm{n}^{\circ}$ 2, Le Conte oriental, 2004-2005, p. 109-124. 
pas fortuit, les deux écrivains se connaissent - plutôt bien, même s'il est difficile « biographiquement » de préciser leurs relations exactes. Mais on pourrait tout aussi bien parler de la Peau d'Âne de Perrault - là encore aucune magie si ce n'est celle où s'origine le pouvoir royal, l'âne faiseur d'Ordure... Référence qui n'est pas non plus absente de Titi.

Enfin : l'intrication du politique, de l'économique et du féerique ${ }^{10}$ trouve une résonance particulière dans/par le concept de "magnificence »: le féerique travaille le concept politico-financier et fait justement éclater l'unité du concept en deux sphères, celle de la grandeur politique et celle de la valeur économique. Au moment où, depuis Vauban, et surtout avec John Law et Jean-François Melon (qui est le correspondant à Paris à la fois de Saint-Hyacinthe et de Montesquieu au début des années $173 \mathrm{O}^{\mathrm{II}}$ ), une nouvelle pensée économique " néo-mercantile » ou " proto-libérale " se met en place, où les questions de la valeur monétaire conventionnelle et de la circulation sont au cœur des débats ${ }^{12}$, il est intéressant d'examiner avec précision comment dans Titi la question de l'économique vient perturber (infléchir mais peut-être aussi contrarier) le projet politique.

La question est donc de comprendre comment, dans Titi, s'élabore une nouvelle conception du politique, étroitement liée à des conditions d'exercice économiques et financières tout en prenant en compte le tricotage opéré par le féerique et déjà largement dénoué et peut-être défait par le conte lui-même.

Car projet politique, il y a, indéniablement - et je commencerai par là.

\section{La fable politique de Titi}

D'abord, on ne peut tout à fait ignorer pour l'Histoire de Titi, l'histoire de Saint-Hyacinthe, ni l'Histoire de la France et de l'Angleterre qui y interfe-rent et font du matériau biographique et contextuel une pièce importante

IO. Yves Citton a développé cette question chez Melon à propos de son conte Mahmoud le Gasnévide (I729) : «Les comptes merveilleux de la finance : confiance et fiction chez Jean-François Melon ", dans Féeries, n 2, Le Conte oriental, 2004-2005, p. I25-160.

II. Elisabeth Carayol, Thémiseul de Saint-Hyacinthe, ouvr. cité, p. I20, note 90.

I2. "Mais l'or et l'argent sont encore devenus insuffisants par l'augmentation continuelle du commerce, et ils ont eu besoin eux-mêmes d'une nouvelle représentation par les billets, lettres de change, banques, etc. ", Melon, Essai politique sur le commerce [ $\mathrm{I}^{\mathrm{re}}$ éd., I734; $2^{\mathrm{e}}$ éd. augm. de 7 chapitres, I736] dans Eugène Daire (éd.), Économistes financiers du XVIII siècle, Paris, Guillaumin, I846, p. $707-836$ et p. 709 . 
du puzzle qui incite à lire le conte précisément comme une fable politique : je donnerai juste quelques uns de ces éléments déterminants ${ }^{13}$.

- Hyacinthe Cordonnier (I684-I746), connu sous le pseudonyme de Thémiseul de Saint-Hyacinthe, a opté, après une carrière d'officier français très brève (quelques mois en 1703-I704) pour une activité d'écrivain polygraphe et engagé en Hollande où il participe au Journal littéraire (entre I7I3 et I722) avec les protestants Van Effen et Van's Gravesande.

- En I7I4, il devient l'auteur d'un livre à sensation, le Chef d'œuvre d'un inconnu, qui récuse toutes les formes d'autorité, à commencer par l'auctorialité pédante, le livre paraissant sous le nom du pédant Mathanasius. C'est un immense succès, qui occupe le $\mathrm{I} 4^{\mathrm{e}}$ rang des ouvrages les plus fréquents du XVIII ${ }^{\mathrm{e}}$ siècle, avant tous les romans ${ }^{14}$. Il semble alors être passé, selon sa biographe, Elisabeth Carayol, d'une demi-indifférence en matière de religion à un " doute actif " ("état actif de doute philosophique ${ }^{15}$ ») et la légèreté dont il fait preuve vis-à-vis des personnages bibliques choque ses collègues protestants.

- De retour en France, après I716 (entre I7I6-I723 puis entre I73II740), il mène une vie mondaine, détient un office dans la maison du Régent et publie des écrits théoriques et politiques (des Entretiens dans lesquels on traite des Entreprises de l'Espagne en 1719, plus tard, en I743, des Recherches Philosophiques), dans la lignée des philosophies du droit naturel (Grotius, Puffendorf, Locke). Dans les années I730, il fréquente le Salon de $\mathrm{M}^{\mathrm{me}}$ de Lambert et rencontre Fontenelle, Montesquieu, Maupertuis, Ramsay... Voltaire avant leur brouille éclatante.

- Mentionnons aussi le «séjour » anglais - plus qu'un séjour, c'est une vraie installation entre I723 et I73I : il s'y marie, fait partie de la Royal Society de Londres (il est l'un des trois parrains exigés pour l'élection de Montesquieu), est tenté par les affaires dans « la librairie », a de gros soucis financiers, la pension accordée à la famille de Saint-Hyacinthe étant, de plus, supprimée par George II.

- En 1736, donc : paraît d'abord un Recueil de divers écrits (de divers auteurs issus du cercle de $\mathrm{M}^{\text {me }}$ de Lambert, Saint-Hyacinthe en est

I3. Empruntés à E. Carayol, ouvr. cité.

I4. Selon l'enquête de Daniel Mornet, "Les enseignements des bibliothèques privées (I750I780) ", dans Revue d'Histoire littéraire de la France, I910, p. 460 et suiv.

I5. Selon E. Carayol, ouvr. cité, p. 52-54. 
l'« éditeur " scientifique), avec une dédicace au prince de Galles, Frederick, fils et héritier du roi d'Angleterre, George II ; dédicace qui est un éloge du type du despotisme éclairé. Puis paraît l'Histoire du Prince Titi (Paris, Vve Pissot, 7 livres en 3 volumes). C'est un succès de

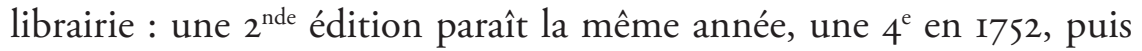
c'est l'édition dans Le Cabinet des fées (vol. 27 et 28, en 1786, qui comprend un $8^{\mathrm{e}}$ livre apocryphe, écrit par Mayer). Paraissent aussi plusieurs traductions anglaises dont une par Elizabeth Stanley (Londres, I736) qui comporte un sous-titre éclairant pour notre propos: The History of Prince Titi, a royal allegory. Par ailleurs, Frederick adopte pour luimême comme surnom "le Prince Titi ${ }^{16}$.

Ainsi, le matériau biographique permet de définir un profil d'auteur polygraphe, journaliste-libraire, engagé (qui a écrit une marqueterie de pièces diverses, mondaines, politiques, philosophiques, romans, nouvelles, contes, essais - ou encore des traductions, celle de Robinson Crusoé) : le souci du politique (de la chose publique, de la lutte contre les préjugés) y est constant, de même que le lien avec des pensées/penseurs de l'histoire politique et économique de premier plan : Fontenelle, Montesquieu, Melon.

À partir de là, la lecture "politique " de Titi apparaît comme une évidence et c'est sans nul doute la lecture qui a été faite à l'époque : en témoigne le titre de la traduction anglaise cité ci-dessus. L'ouvrage a d'abord été lu comme une satire de George II et de l'absolutisme et un éloge du despotisme éclairé, via la figure du prince héritier FrédérickTiti :

Un ensemble de traits se rapporte en effet à la situation anglaise de l'époque : un roi et une reine, peu populaires et avares, sont sur le trône. La reine Trip[asse], comme Caroline en Angleterre, est par ailleurs une femme intelligente et cultivée, qui prend sur son époux un grand ascendant au point de lui imposer les favoris politiques de son choix. L'opposition, de son côté, met son espérance dans le Prince Titi, abrégé de toutes les vertus, comme elle se regroupe en Angleterre autour du prince de Galles ${ }^{17}$.

I6. Rapidement, un aperçu de la fin de sa vie : un retour en Hollande, après 1742, une nouvelle participation dans un périodique Le Fantasque où, en 1745 , huit Lettres persanes de Montesquieu sont insérées, ce qui atteste sans doute la persistance de la liaison entre les deux hommes.

I7. E. Carayol, ouvr. cité, p. I49. 
Les trois volumes sont même appréciés à l'époque à l'aune du " sérieux " du propos : le premier est taxé de "badin ", les deux suivants, plus graves, sont plus appréciés ${ }^{18}$.

Aussi, l'intrigue de Titi se présente-t-elle très clairement comme une fable politique $^{\text {19 }}$; la structuration du conte-roman s'effectue autour de la quête et de l'exercice du pouvoir selon deux grandes séquences : la quête du royaume ou l'avènement à la couronne : (livres I à 3) et l'établissement de la paix, l'art de gouverner (livres 4 à 7 ). La question de la fin mérite par ailleurs d'être posée et met en lumière justement le projet presque uniquement politique de l'auteur ; le dénouement chez Saint-Hyacinthe est en effet strictement et sèchement politique : le conte s'achève après la récupération de la dernière province. Alors que le dénouement proposé par Mayer pour le livre 8 apocryphe, avec son quadruple mariage, est plus conforme aux codes romanesques ou féeriques - la fameuse " conclusion nécessaire " mentionnée à la fin de l'édition du Cabinet des fées ${ }^{20}$.

Par ailleurs, la part du politique, dans tout l'immense conte est extrêmement développée : presque toutes les questions politiques de l'époque sont présentes et même problématiques, c'est-à-dire qu'elles donnent lieu à la fois à développement et à questionnement. On peut en énumérer quelques-unes : celles des querelles de succession, de la diplomatie et des guerres, de la justice et du judiciaire, des relations entre les provinces du royaume, entre la Capitale et les marges, des privilèges nobiliaires, et aussi de très nombreuses questions économiques ayant trait à la misère du peuple, les finances de l'État, la spéculation, le droit de propriété, etc.

Mais, bien sûr, ce qui dirige et modélise ces questions, c'est le portrait - et aussi le regard - du prince :

[...] Titi, en plus de ses aspects féeriques et polémiques veut être un bréviaire de bon gouvernement dans l'optique philosophique [...]. On trouve dans le courant du récit d'innombrables maximes morales sur les devoirs des princes et leur mise en œuvre par le vertueux Titi qui visite incognito son royaume pour se rendre compte lui-même de l'état de son peuple, qui s'efforce de rendre la prospérité à son pays ruiné par la guerre, de gouverner en tout selon la raison et la justice ${ }^{2 r}$.

Je ne détaillerai pas les éléments constitutifs du modèle du despotisme éclairé - c'est la seule lecture qui, à ma connaissance, a été menée sur Titi,

\footnotetext{
I8. Voir E. Carayol, ouvr. cité, p. I48-I5I.

19. Voir en Annexe le résumé du conte-roman.

20. Voir supra, note 2.

2I. E. Carayol, ouvr. cité, p. I50.
} 
et je souhaiterais ici plutôt en proposer une autre. En guise de justification, je soulignerai un point : l'entrelacement du réflexif et du fictif souligné par le texte lui-même à plusieurs reprises. Par exemple : l'expérience insulaire des amants Titi et Bibi (au Livre $3^{e}$ ) et le modèle utopique de société qui en découle sont, au moment de leur possible application (au Livre 6 $6^{\circ}$ par Titi, devenu roi, longuement désavoués :

[...] pour nous [Bibi et Titi] faire voir combien elle [la raison] pouvait contribuer à son [l'homme] bonheur, nous nous imaginâmes dans notre île, une société de gens raisonnables ; nous leur faisions faire tout ce que la bonté du cœur et ce que l'humanité en exigeaient ; nous étions charmés de la concorde, des services mutuels, de la bonne foi, des plaisirs ; en un mot, du bonheur qui régnait parmi eux : nous voulûmes sur ce fondement établir un gouvernement parfait ; mais nous nous aperçûmes aussitôt que nous étions dans l'erreur, et qu'entre des gens raisonnables, il n'y avait point de gouvernement à établir, puisque l'établissement d'un gouvernement n'est que pour faire faire aux hommes déraisonnables ce que la raison veut qu'ils fassent. Les lois ne sont faites que pour réprimer leurs injustices, leurs passions désordonnées, et les magistrats ne sont établis que pour faire observer ces lois. Si nous peuplons cette île de gens raisonnables, disions-nous, nous ne nous donnerons point de sujets, nous ne nous donnerons que des amis, et nous aurons fait tout d'un coup ce que les bons princes se trouveraient trop heureux de pouvoir faire, si la chose était possible. Mais comme ce n'était que l'ouvrage de notre imagination, continua Titi, nous ne nous occupâmes pas longtemps de cette agréable chimère. Nous passâmes à la considération des hommes tels qu'ils sont, ayant besoin de lois et de magistrats pour remédier à leur extravagance et à leur corruption, et nous prîmes ce royaume et quelques-uns des états voisins pour le plan sur lequel nous devions travailler. Nous parcourûmes les différents arrangements et les divers désordres qui s'y trouvent, et nous cherchâmes avec soin les moyens d'assurer ou de perfectionner les uns, et de remédier aux autres, conformément aux principes de justice pris dans la nature des choses mêmes. Je m'y appliquais avec d'autant plus d'attention, ajouta Titi, qu'il se pouvait faire que j'aurais un jour besoin d'être muni des principes nécessaires à un bon gouvernement. Nous les recherchâmes donc avec exactitude, et nous tirâmes des règles applicables à tout ce qui convient à une société civile ${ }^{22}$.

On reconnaît l'argumentation du vieillard Troglodyte chez Montesquieu (la Lettre I4 des Lettres persanes) : " vous aimez mieux être soumis à un prince, et obéir à ses lois moins rigides que vos mœurs ». Celle-ci contribue à mettre à distance le modèle purement rationnel du " despote éclairé " (il ne suffit pas d'être raisonnable et d'avoir un "plan " juste) et à interroger le lien entre fable et politique, entre théorie et pratique aussi, entre réflexion et expérience : Titi relègue l'expérience insulaire à un statut idéal, l'utopie, où le tableau insulaire n'a donc plus statut de fable allé-

22. Histoire du Prince Titi, ouvr. cité, Livre Sixième, tome 28, p. 260-26I. 
gorique ( il y a de certaines vérités qu’il ne suffit pas de persuader mais qu'il faut encore faire sentir ", dit Usbek en préliminaire à l'apologue des Troglodytes, Lettre in des Lettres Persanes) et s'écarte alors, semble-t-il, de la poétique de Montesquieu. Mais, en fait, il s'agit seulement d'opposer deux modèles d'expérience : l'un, hors-monde, l'autre, dans le monde.

La fiction de Titi a bien alors statut de fable : c'est bien le parcours du héros - et non seulement les multiples " maximes " de bon gouvernement qui essaiment le texte - qui fait sens, politique et philosophique. Pas de théorie sans expérience préalable mais aussi, la prescience d'une dialectique nécessaire entre théorie et pratique. Ainsi, au Livre $4^{e}$, à propos des Mémoires demandés aux personnels compétents :

Ce n'est pas que Titi voulut se conduire par les avis qu'on lui donnerait ; il avait son plan ; il voulait seulement le perfectionner encore avant que de le mettre en exécution, et il croyait que ces mémoires pourraient y contribuer. D’ailleurs, il était persuadé que ceux qui n'ont appris les affaires que par les emplois qu'ils ont eus, ne sont pas toujours les gens les plus propres à en bien juger, parce qu'ils ont des préjugés d'usage qu'ils prennent pour des règles nécessaires, quoiqu'elles n'aient souvent qu'un fondement abusif ; mais il croyait aussi que les gens du métier pouvaient apprendre aux autres plusieurs choses que la spéculation ne découvrirait jamais, ou du moins à quoi elle ne parviendrait que très difficilement. C'est pourquoi il voulait toujours premièrement juger des choses d'une manière spéculative, et vérifier, étendre ou rectifier ensuite les raisonnements par l'expérience. Il donna quatre mois pour dresser les mémoires, et jusqu'à ce temps-là il ne fit aucun changement dans l'administration des affaires ${ }^{23}$.

Enfin, un dernier point concernant l'élaboration de la fable politique : le modèle "idéal " (qui n’est donc pas théorique) ne recoupe pas uniquement la figure de Titi mais se construit tout au long du conte-roman par confrontation de plusieurs portraits princiers, le plus évident, outre celui de Titi, étant celui de Forteserre, roi conquérant, au patronyme construit sur le modèle shakespearien de Fortinbras ${ }^{24}$, roi également susceptible, colérique, libertin mais qui possède, de façon corollaire, toutes les qualités du modèle héroïque et féodal auquel il semble appartenir, force, loyauté, vertu/courage, franchise... Le parcours du prince Titi est d'ailleurs celui d'une alliance avec Forteserre : pendant tout le $6^{\mathrm{e}}$ livre, les deux princes voyagent et élaborent ensemble un modèle de gouvernement. Ils

\footnotetext{
23. Ouvr. cité, tome 28, p. 39-40.

24. La reprise du Fortinbras de Hamlet a déjà été faite par Hamilton chez qui il était devenu "Fortimbras à la Grand-Bouche, roi de Danemark ", dans Les Quatre Facardins publiés en 1730 (Contes, éd. critique par Jean-François Perrin, Paris, Champion, "B.G.F. ", vol. I6, 2008, p. 574, note II7).
} 
échangent, alors, partiellement leurs attributions respectives : Forteserre apprend à dominer sa colère, Titi, métamorphosé en aigle, sauve la vie de Bibi en tuant un ennemi "d'un coup de serre ${ }^{25}$ "; le choix du terme ne pouvant guère être fortuit dans un récit où l'invention onomastique est particulièrement riche (voir, parmi des dizaines d'exemples possibles, les noms enfantins, garants d'une "ingénuité ", de Titi et $\mathrm{Bibi}^{26}$, du Palais conjugal Bititibi où l'élément féminin enlace le masculin en un renversement idéologique notable, ou encore les noms transparents des " fidèles » et féodaux de Titi, Fullfoi, Frycore, Hopevaine,Vaervir). Le roi Forteserre est donc bien celui qui à la place d'une tête présente une serre - et une forte serre, comme l'on parle de forte tête. Mais il y a également un troisième modèle, utopique ou pastoral, représenté par (feu) le roi de Félicie et ses lois très libérales sur le mariage conçu comme pacte de consentement mutuel et civil, à durée déterminée :

Il me semble que dans un pays où le mariage n'est considéré que comme un contrat civil, on devrait établir les lois du prince de Félicie. Il permet de s'unir aux conditions qu'il plait naturellement aux contractants de s'accorder. En conséquence de quoi ils peuvent s'engager l'un à l'autre pour un temps ou pour toujours. Se permettre plusieurs femmes ou plusieurs maris; car il a trouvé juste que les droits fussent égaux ; stipuler en cas de séparation des dédommagements pour l'un ou pour l'autre, régler l'état des enfants ${ }^{27}$.

Cette confrontation des modèles trouve ainsi sa plus claire représentation dans le déplacement du lieu du pouvoir traditionnel (la Capitale) et l'omniprésence de la "frontière " comme lieu de l'exercice du pouvoir : deux espaces coexistent sans cesse dans Titi, la Capitale et les marges du royaume ${ }^{28}$. C'est sur et par la frontière que se renouvellent le pouvoir politique (confrontation et alliance avec le voisin Forteserre) et le sang royal (Bibi vit à la frontière du royaume et n'est pas noble); et c'est la frontière qui est l'espace dévolu à la célébration royale (Palais de Bititibi et fête princière). Enfin, c'est lors de la décisive et très longue (une centaine de pages) séquence de la fête royale qui réunit tous les protagonistes (les rois, la fée, les princesses, l'Éveillé) que se débat justement le modèle politique et économique devant servir de fondement au gouvernement de Titi : c'est

25. Ouvr. cité, Livre Second, tome 27, p. 430.

26. «Biby " est également un nom de serin dans le Dauphin de $\mathrm{M}^{\mathrm{me}}$ D’Aulnoy.

27. Ouvr. cité, Livre Cinquième, tome 28, p. 132.

28. J'ai déjà développé ce point dans une autre étude dont je reprends brièvement les quelques lignes qui suivent et à laquelle je renvoie pour de plus amples conclusions, "Saint-Hyacinthe : écrire la marge ", dans C. Bahier-Porte et R. Jomand-Baudry (éd.), Écrire en mineur au XVIII siècle, Paris, Desjonquères, 2009, p. 40-50. 
là que Forteserre apprend à maîtriser sa colère, à revenir sur ses préjugés nobiliaires, là enfin, que se produit la scène clef de l'inversion de la valeur fiduciaire. Forteserre prétend transformer la fête royale en une fête égalitaire en instaurant la règle d'un demi-ginguet d'amende à qui prononcerait le mot de "Majesté » mais la fée Diamantine, sous les traits d'une vieille femme, joue à transgresser la règle et finit par multiplier les ginguets de telle sorte que ni la monnaie ni la souveraineté n'ont plus cours.

Aussi, ce qui ressort plus nettement : c'est l'interaction du politique et de l'économique, c'est en effet sans cesse la question des finances du royaume qui ressurgit lors d'épisodes décisifs (la séquence de la réunion d' "États généraux ", la politique somptuaire liée à la célébration royale) et qui détermine la question politique. Mais la question économique dans le conte n'est pas séparable de la question de l'économie du conte (ou de la merveille) ; plusieurs épisodes, en plus de celui du repas " égalitaire " de la fête royale, l'éclairent particulièrement : la situation initiale autour des portraits contrastés d'avaricieux et de prodigues, les fruits magiques donnés par Diamantine (et la figure de Diamantine en général), les bourses inépuisables, enfin, la construction du palais de Bititibi. La complexité de la signification (qui n'est donc pas, on le voit, de l'ordre d'un quelconque " message ») politique de Titi repose donc aussi/surtout sur l'usage qui est fait du matériau féerique et sur ce que j'ai appelé le «tricotage » de l'économie et du merveilleux.

\section{Système économique et logique féerique}

D'abord : rappelons que le modèle politique repose tout entier sur un modèle économique à inventer. C'est la prise de conscience de la misère du peuple (des campagnes), des ravages de la guerre (avec un rendu des realia saisissant), qui conduit à penser un modèle politique ; pas seulement par souci de justice sociale (même si l'idée est présente) mais aussi par «calcul » politique : le meilleur État étant le plus prospère, il faut penser les conditions économiques susceptibles de favoriser cette prospérité. Le modèle proposé est clairement mercantile et rejoint en cela les propos d'un Boisguilbert ou d'un Melon ${ }^{29}$ : c'est l'abondance de valeur produite et la circulation de l'argent qui comptent ; d'où chez Melon une comparaison entre l'actionnaire et le rentier, à la défaveur (malgré les

29. Voir Jean-Yves Grenier, Histoire de la pensée économique et politique de la France d'Ancien Régime, Paris, Hachette, 2007, notamment les chapitres 5 et 6. 
précautions oratoires) de ce dernier ${ }^{30}$. De même, dans Titi, entre les trois modalités de l'avarice, de la dépense magnifique et de la spéculation, on peut facilement établir une hiérarchie : en tête des valeurs les plus prisées vient la dépense, sur le modèle des fêtes du couronnement où, à la lettre, on jette l'argent par les fenêtres ${ }^{31}$. Ensuite, arrive la spéculation, sur le modèle du fermier qui spécule sur ses 13 arpents (soit environ 5-6 hectares au maximum) : elle est condamnée moralement mais ni économiquement ni narrativement parlant puisque le récit se contente de la contourner ; seule l'avarice est absolument condamnée.

Tout le roman peut d'ailleurs se lire selon ce modèle économique et comme un circuit financier : qu'on considère maintenant quelques uns des épisodes mentionnés ci-dessus; et d'abord, la situation initiale.

À l'origine - du récit, du royaume : l'avarice absolue. L'alliance de Ginguet, roi de Magnastrück, et de Tripalle, pose les fondements du royaume :

Il choisit Tripalle, par ce qu’on lui avait marqué qu’elle était si bonne ménagère, qu'elle ne se faisait faire tous les ans qu'un habit, et même qu'elle le faisait si bien rajuster, qu'il lui servait quelquefois dix-huit mois ou deux ans ; qu'elle filait à merveille, et que quand elle avait beaucoup de fil, elle en faisait une loterie, que les seigneurs et les dames de la cour s'empressaient à remplir pour lui plaire. [...] L'argent de cette loterie était un revenu si considérable, qu'il n'y avait point de livre de fil qui ne lui rapportât ainsi plus de quatre mille florins $[\ldots]^{32}$.

La loterie n'est pas qu'un jeu de hasard : elle peut aussi être un moyen économique. André-François de Brancas-Villeneuve publie ainsi en 1754 une curieuse utopie économiste, l'Histoire ou Police du Royaume de Gala et propose, comme méthode de lever les impôts et d'encourager le commerce, une loterie forcée pour la recette des droits d'entrée dans la capitale de Gala ${ }^{33}$. Elle a également été utilisée, notamment par les Romains, comme outil de prodigalité (grâce à la gratuité des billets) dans le cadre des saturnales. Le commentaire qu'en fournit Jaucourt pour l'Encyclopédie

30. Melon, Essai politique sur le commerce, ouvr. cité, p. 729.

3I. "On exagère peut-être, mais on dit qu'il [un avare] jeta ainsi plus de deux cens mille écus. Son exemple fut suivi de tous ceux qui avoient de l'argent chez eux; les uns plus, les autres moins. Les ginguets d'or et d'argent, toutes sortes d'espèce de monnaie, pleuvaient dans les rues; ceux qui n'avoient point d'argent monnayé, y jetèrent leur vaisselle. " (Histoire du Prince Titi, ouvr. cité, tome 28 , p. IO)

32. Ouvr. cité, tome 27, p. 330-33I.

33. André-François de Brancas-Villeneuve, Histoire ou Police du Royaume de Gala, Londres [Paris], I754. 
de Diderot et d'Alembert dans la seconde moitié du siècle dit assez en quelle piètre estime les Lumières la tenaient :

Enfin en 1685 Louis XIV renouvela dans ce royaume la mémoire des anciennes loteries romaines : il en fit une fort brillante au sujet du mariage de sa fille avec M. le Duc. Il établit dans le salon de Marly quatre boutiques remplies de ce que l'industrie des ouvriers de Paris avait produit de plus riche et de plus recherché. Les dames et les hommes nommés du voyage, tirèrent au sort les bijoux dont ces boutiques étaient garnies. La fête de ce prince était sans doute très galante, et même à ce que prétend M. de Voltaire, supérieure en ce genre à celle des empereurs romains. Mais si cette ingénieuse galanterie du monarque, si cette somptuosité, si les plaisirs magnifiques de sa cour eussent insulté à la misère du peuple, de quel oeil les regarderions-nous ${ }^{34}$ ?

La loterie apparaît aussi, dans le conte de Titi, comme un moyen économique à part entière, mais un moyen économique d'asservissement à l'augmentation du Capital d'un seul. Le travail produit par la reine très " travailleuse " ("Tripalle» de Tripalium ?) ne sert que sa propre abondance au lieu de produire le circuit nécessaire à l'abondance des autres. La loterie est le symbole même de cette immoralité du fondement économique du royaume : "Pourquoi faut-il que ceux dont le travail produit l'abondance des autres, ne jouissent pas des biens que leur travail procure ${ }^{35}$ ? ", s'exclame Titi à la fin de son parcours d'apprentissage. La loterie, à l'origine de la magnificence royale, peut également évoquer l'Âne de Perrault : sauf que la reine travaille dur pour produire cette ressource " merveilleuse ", sauf aussi qu'elle travaille justement au lieu d'enfanter. Elle travaille au lieu d'être " en travail », elle transforme le cycle naturel en circuit financier ; seuls deux enfants, comme les deux faces (pile et face) d'une même pièce de monnaie voient le jour : "sa naissance [celle de Titi, l'aîné] fit craindre au roi et à la reine trop de fécondité, ils trouvaient qu'il était venu trop vite ; néanmoins la reine eut encore plusieurs autres enfants qui moururent tous en bas âge, excepté le dernier de tous ${ }^{36}$ ". L'avarice est bien une structure économico-politique : elle conduit à la stérilité (des époux) et à la misère (du peuple).

Le règne de Ginguet est d'ailleurs scellé emblématiquement par la création d'une monnaie à l'image du roi (comme le louis pour Louis), mais bien sûr, la proposition est réversible et c'est tout aussi bien le roi qui est à l'image de sa monnaie, qui devient même sa monnaie, un roi-monnaie : le

34. Article «Loteries " (des Romains), Encyclopédie de Diderot et d'Alembert. L'article est du chevalier de Jaucourt.

35. Histoire du Prince Titi, ouvr. cité, Livre Sixième, tome 28, p. $25 \mathrm{I}$.

36. Ouvr. cité, Livre Premier, tome 27, p. 332. 
ginguet (qui peut être d'or ou d'argent, demi-ginguet, etc.) est le symbole de cette royauté sans valeur ou qui se trompe sur la valeur, qui confond les valeurs : un vin "ginguet ", renseignent Furetière, l'Académie puis Littré, désigne un petit vin vert et mauvais, qui donne par métonymie la " guinguette» ("petit cabaret où l'on boit du petit vin appelé guinguet, du mot ginguet, étroit, serré, mince $\left.{ }^{37} »\right)$ et par extension, l'adjectif s'applique à tout ce qui a "peu de valeur " et " peu de force".

L'épisode suivant, celui des fruits magiques donnés par la fée Diamantine, constitue alors le véritable embrayeur du récit : d'abord, ils soulignent, sous l'apparence fastueuse, la fausse magnificence, l'avarice mesquine du couple de souverains. Les "nèfles » et autres noix se transforment en diamants mais lors de la réception royale du souverain étranger, redeviennent des nèfles et déclenchent la guerre. Le don magique signale donc clairement que suivant la métaphore courante, Tripalle et Ginguet ne sont que des roi et reine de "nèfles " (Furetière atteste le sens « cela me coûte de bon argent, je ne l'ay pas eu pour des nèfles ") et aussi que l'avarice engage une dynamique de guerre et de misère. La stérilité de la reine devient contagieuse et provoque la stérilité du peuple : on peut notamment retenir l'image saisissante d'une paysanne " noire et desséchée ", à la " mamelle aride".

Comme ils [Titi et Forteserre] parlaient ainsi, ils aperçurent sous un arbre, près du chemin, un enfant en maillot que sa mère avait posé sur un casaquin qu'elle avait quitté pour mieux travailler. Elle bêchait un champ à l'ardeur du soleil, qui, quoiqu'il fût haut, n’avait pas la force de sécher la chemise que la sueur collait sur le dos de cette pauvre femme. Bonne mère, lui dit le roi, pourquoi faut-il que vous travailliez ainsi vous-même ? Pourquoi, répondit cette femme, sans quitter son travail ? C'est qu'il faut que cet ouvrage se fasse, et que si je ne le fais, il ne se fera pas tout seul. Mais il me semble, ajouta le roi, que bêcher ainsi la terre serait l'ouvrage de votre mari, et non pas le vôtre. Hélas, dit-elle, il est dans son lit, mon pauvre homme! et c'est pour avoir de quoi lui faire du bouillon, que je me hâte de finir aujourd'hui ce morceau de terre ; il l'avait entrepris, et il n'a pu l'achever. En disant ces mots, cette femme regarda mieux à qui elle parlait, et se dressant sur sa bêche, elle parut plutôt un spectre, qu'une créature humaine, tant elle était noire et desséchée. Mais, poursuivit le roi, n'avez-vous pas quelque enfant, ou quelque voisin qui puisse faire pour vous ce que vous faites, ou du moins qui puisse vous y aider ? Mes voisins, monsieur, répondit la femme, ils ont assez de mal à travailler comme il faut pour eux ; et pour des enfants, mon homme en avait un qui aurait bien fait cet ouvrage, car il était bon

37. François Noël et L.-J. Carpentier, Philologie française ou Dictionnaire étymologique, critique, historique, anecdotique, littéraire... pour servir à l'histoire de la langue française, Paris, le Normant Père, I83I, p. 728. Apparemment, " ginguet " se prononçait et même souvent s'écrivait "guinguet". 
travailleur ; mais on l'a pris pour l'emmener à la guerre, et il n'en est pas revenu, le pauvre garçon. Elle vint alors boire dans une cruche, de l'eau qu'elle y avait apportée. Elle s'assit sous l'arbre, prit son enfant, et lui donna à sucer une mamelle aride ${ }^{38}$.

Après les fruits magiques qui lancent le récit, il y a les innombrables bourses magiques toujours données par Diamantine, la grande trésorière du royaume, dont le nom évoque une monnaie forte, en opposition à la monnaie faible du " ginguet " : ces bourses inépuisables ont de multiples fonctions dans le récit, elles permettent de financer les guerres et les réjouissances, de suppléer sans cesse au défaut d'argent de Titi et à la misère du peuple, surtout, elles constituent, avec la fée Diamantine, dont elles sont les métonymies, l'image même du circuit économique déficient du royaume. Par exemple, au début du récit, Titi, pour faire la charité à une vieille femme (qui n'est autre que Diamantine) doit emprunter à son serviteur, l'Éveillé, qui lui-même emprunte la somme requise à son père : le père reçoit alors en retour la première bourse inépuisable et à l'autre terme du récit (au Livre 6 $\sigma^{c}$, le serviteur se voit récompensé d'un royaume : "Qui croirait [dit l'Éveillé], que quatre sols et demi, prêtés par un pauvre page, pour le soulagement d'une pauvre femme, eussent pu procurer à ce page une souveraineté 39 ? " Dès lors, le Prince n'est pas hors du circuit financier, il participe, au même titre que ses sujets, à la circulation monétaire : il est, selon le mot du «fondateur » de l'économie politique du début du XvıII ${ }^{\mathrm{e}}$ siècle, Boisguilbert, à la fois « vendeur et acheteur et vice versa ${ }^{40}$ ». Ce que met en branle Diamantine et ses bourses magiques, c'est donc bien un véritable réseau financier et commercial, un " circuit " économique, système interdépendant dont on sait qu'il est au cœur des nouvelles théories économiques de Boisguilbert et Melon ${ }^{4 \mathrm{I}}$.

Et c'est là que ressurgit la question de l'intrication du féerique, de l'économique et du politique : que penser, en effet, de ce ressort magique de l'argent dans Titi comme fondation même du juste gouvernement? L'économique et le politique ne s'élaborent, de fait, qu'à partir de la merveille : la bourse magique est d'ailleurs presque la seule merveille (excepté les métamorphoses et le don d'invisibilité) du récit ; l'objet merveilleux par excellence dans Titi, c'est le ginguet; les autres objets merveilleux

38. Histoire du Prince Titi, ouvr. cité, Livre Sixième, tome 28, p. $25 \mathrm{I}-252$.

39. Ibid., tome 28, p. I42.

40. Pierre Le Pesant de Boisguilbert, Dissertation de la nature des richesses, de l'argent et des tributs, s.l. s.d. [1707], réed. dans Eugène Daire (éd.), Économistes financiers du XVIII siècle, ouvr. cité, chapitre 4, p. 383.

4I. Voir Jean-Yves Grenier, Histoire de la pensée économique et politique de la France d'Ancien Régime, ouvr. cité. 
(comme le sabot pour mesurer la fidélité ou le gobelet pour calmer la colère) sont de fausses merveilles. S'agit-il, alors, d'une mise en perspective du politique et de l'économique par le féerique, le politique étant ainsi pris dans le miroir des fées ? Quel enjeu cela peut-il avoir ? L'idée d'un ressort magique de tout pouvoir?

Peut-être pas, car l'économie nouvelle est justement conçue comme une interaction d'effets aux ressorts sinon cachés, du moins difficiles à établir et somme toute peu importants : selon la pensée de Boisguilbert dans sa Dissertation de la nature des richesses, de l'argent et des tributs ${ }^{42}$, ce qu'il faut, désormais, comprendre ce sont précisément les effets et non les ressorts, ce qu'il importe de dénombrer, ce sont les agents extrêmement variables qui entrent dans l'interdépendance du "circuit économique ». Pour Boisguilbert, l'argent est une vieille "idole », l'argent est " criminel ${ }^{43}$ "; on ne doit pas confondre ce qui permet l'échange et ce qui produit la richesse : ainsi, « ce n'est pas avec de l'argent, en réalité, que le souverain entretient les armées de terre ou de mer, et rétribue tous les services publics ${ }^{44}$ ". À partir de là, peu importe d'où provient l'argent, c'est sa bonne administration qui compte : d'où, peut-être, les très longs atermoiements de Titi au Livre $5^{e}$ pour décider du prix juste qu'il convient de donner aux propriétaires terriens d'une parcelle qu'il veut acheter pour construire son Palais. Il s'agit justement d'obtenir ce " prix de proportion " établi par Boisguilbert, c'est-à-dire un juste partage du profit entre acheteur et vendeur ${ }^{45}$ : la longue négociation prend ainsi successivement en compte la "valeur ordinaire " du terrain, le léger supplément qui convient pour satisfaire les vendeurs qui ne souhaitaient pas initialement s'en défaire, ou pour les " dédommager du dérangement que cela peut [leur] causer ${ }^{46}$ ", puis le prix « exorbitant » demandé par l'un des vendeurs qui intègre dans le prix réclamé la plus-value du terrain une fois le Palais construit. Le prix oscille alors entre " rien » (" moi, dit un troisième, si on m'en croit, nous les donnerons pour rien $\left.{ }^{47} »\right)$, I 200 et 40000 ginguets d'argent. Titi est prêt alors, par équité, à payer à chaque vendeur la somme extraordinaire demandée par un seul mais c'est alors l'État qu'il mettrait en défaut puisqu'il s'agit de sa dépense privée et non d'une publique. La

42. Boisguilbert, Dissertation... dans E. Daire (éd.), Économistes financiers du XVIII ${ }^{e}$ siècle, ouvr. cité, p. 372-407.

43. Ibid., p. 489-490.

44. Ibid., chapitre vi, p. 396.

45. Ibid., p. 383.

46. Histoire du Prince Titi, t. 28, p. 182.

47. Ibid. 
solution trouvée est alors politique et non plus économique : il laisse la parcelle intacte et l'entoure d'une muraille : «Ainsi je n'ai pas tort de vous dire que ce sera le plus bel endroit du parc, puisque ce sera une marque de modération des rois, et de leur attention à ne pas violer ce qu'on appelle la propriété de leurs sujets ${ }^{48}$."

Il s'agit donc bien, en définitive, d'une leçon de magie économique mais pas au sens où les savoirs d'économie politique seraient tout à fait oblitérés ou détournés par la féerie : les ressources merveilleuses du royaume ne sont que les conditions de l'expérimentation d'une pensée et d'une théorie nouvelle en train de s'émanciper. Ainsi, le conte entrelace bien les nouveaux savoirs financiers et économiques et l'économie narrative et merveilleuse : par la féerie (significativement par Diamantine plus que par Titi), il présente, expérimente et modélise des savoirs en cours de constitution. La "leçon » de féerie économico-politique est alors assez différente de celle attendue du despotisme éclairé : ainsi, la leçon du conte n'est, bien entendu, pas celle de la leçon dans le conte. J'en retiendrai, éventuellement, trois propositions :

- Titi marque l'autonomisation et la laïcisation de l'économique, la prééminence nouvelle de l'économique comme fondement du politique ;

- mais aussi, simultanément, Titi indique les limites d'une pensée économique encore impensée, en train se s'élaborer, la féerie sert à y énoncer l'impensable et à en formuler une première expérimentation ;

- enfin, Titi prolonge et approfondit la réflexion sur l'économie par une mise en question de la notion de valeur : c'est aussi un texte sur la manipulation, qui multiplie les falsifications de toutes sortes, les procès truqués, les faux et usages de faux, fausses lettres, fausses signatures, truquage des "minutes » du conseil royal, etc. ${ }^{49}$. Et la tricherie n'est pas le seul fait du " mauvais couple royal » Ginguet/ Tripalle, mais elle participe aussi de la leçon du "bien gouverner" apprise tout au long de ce long conte-roman d'éducation par Titi : c'est ainsi qu'il instrumentalise l'Éveillé (devenu l'homme à tout faire, la "mouche » et le financier du royaume) ou qu'il détourne l'économique au profit du politique et même de la politique (lors de

48. Ibid., p. 203.

49. Notamment à la fin du Livre Second, ouvr. cité, tome 27, p. 453 et suiv. 
l'affaire de la muraille du palais de Bititibi). Ce qui dit la leçon de magie féerique et que ne disent pas (encore) les savoirs économicopolitiques, c'est que la politique est affaire non plus (ou plus seulement) de représentation (c'était la grande leçon louis-quatorzienne), mais de médiation/médiatisation.

\section{ANNEXE}

\section{Résumé de l'Histoire du Prince Titi de Thémiseul de Saint-Hyacinthe}

\section{Livres I à 3 : l'avènement de Titi à la couronne}

- Incipit : "Ronoby, roi de Magnastrück, était si juste que ses sujets étaient heureux ; aussi lui donnèrent-ils le surnom de Bon. Il tomba malade, tout le royaume fut alarmé. Les médecins firent de leur mieux, mais quoiqu'ils fussent les meilleurs qu'on pût trouver, ils étaient trop ignorants pour le guérir. Ce bon prince mourut. Il n'avait qu'un fils âgé de vingt-trois ans; on le nommait le prince Ginguet. Son père aurait bien voulu le déshériter ; car Ginguet était hautain, farouche et avare. Mais les lois du pays rendaient la couronne héréditaire, un testament n'y pouvait rien changer. Ginguet monta de plein droit sur le trône après la mort de son père. "

- Situation initiale : le roi Ginguet choisit une épouse conforme à son caractère (avare), Tripalle [Tripasse dans la $\mathrm{I}^{\mathrm{re}}$ édition de $\mathrm{I736}$ ], ils ont, neuf mois après le mariage, un premier enfant, "nommé Titi, à cause de sa mine aimable ", de caractère opposé à ses parents, doux, aimable, généreux. Puis, après la mort de plusieurs enfants en bas âge, un deuxième et dernier enfant qui survit, Triptillon. Le couple haït autant Titi qu'il aime son cadet.

- Élément perturbateur : dix-huit années passent. Titi se rend à la chasse avec son écuyer nommé l'Éveillé : ils renversent légèrement une vieille femme, puis la secourent. En récompense, elle leur donne des noisettes, des noix et des nèfles, et plus tard un œuf, qui, à la cour, se transforment en diamants. Titi n'ayant pas d'argent (à cause de l'avarice du couple royal) sur lui, emprunte à son écuyer (qui

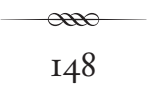


demande lui-même à son père l'argent) quatre écus pour la convalescence de la vieille femme. L'écuyer se fait appeler désormais « le page aux vieilles », la vieille femme est une fée qui s'appelle Diamantine. Elle fait, en retour, un double don au page : le don d'invisibilité et une bourse magique qui ne s'épuise jamais, pour son père. L'Éveillé devient ainsi la "mouche » de la Cour : il informe le Prince Titi des entretiens secrets du couple royal et des ministres.

- La guerre : le roi et la reine s'emparent des diamants et les font coudre sur leurs habits de cérémonie à l'arrivée d'une Ambassade du roi voisin, Forteserre. Les diamants redeviennent des fruits et l'affront déclenche la guerre. Titi est nommé général en chef de l'armée et a auprès de lui quatre fidèles seigneurs, les princes de Fullfoi, de Frycore, de Hopevaine et le duc de Vaervir. Titi se rend sur les frontières du royaume, y bâtit une forteresse (Fort-Titi) et, en attendant les troupes de Forteserre, tombe amoureux d'une jeune roturière, Bibi. Il promet de l'épouser lorsqu'il sera roi et de ne pas la séduire jusque-là : la fée Diamantine intervient et accorde aux amants le don de métamorphose animale, ce qui leur permet, au fil des aventures, d'échapper aux poursuites et de se retrouver discrètement. Titi gagne la guerre contre Forteserre mais demande au roi, clémence pour le roi ennemi, et amnistie pour son peuple. Le couple royal veut faire arrêter Titi, Diamantine propose à celui-ci son aide pour renverser le pouvoir, mais, vertueux, il préfère s'enfuir.

- L'exil : pendant deux années, errance et voyage des amants Titi et Bibi (à l'aide de multiples métamorphoses, moucheron, aigle, etc.). Titi, incognito, parcourt le royaume pour s'instruire de toutes les injustices. Épisode d' " utopie » insulaire (ou plutôt de robinsonnade).

- L'accession au trône : Ginguet meurt (d'apoplexie), la reine entend faire couronner Triptillon (sous le titre de Triptillon $\mathrm{I}^{\mathrm{er}}$ ) mais les quatre seigneurs fidèles à Titi entrent en opposition et la reine, avec son fils, prend la fuite et fait se révolter une province éloignée du royaume contre le pouvoir central. Titi reprend figure humaine et se fait reconnaître roi.

\section{Livres 4 à $8:$ l'art de gouverner}

- Le rétablissement de la paix dans le royaume : Titi fait réunir, sur le modèle des États généraux d'Ancien régime, une assemblée chargée, 
par département de compétence, de "donner des mémoires sur ce qui se faisait, et sur ce qu'il y avait à faire pour le mieux ». Il donne quatre mois de délai et attend le résultat de l'enquête pour commencer à agir au redressement économique du pays ruiné par la guerre. Il instaure un droit de remontrance, rétablit l'ordre, gouverne selon la justice et la vertu.

- La fête royale : une fête magnifique réunissant les deux cours de Titi et de Forteserre est organisée sur la frontière, au cours de laquelle réapparaît Diamantine sous les traits d'une vieille femme : elle court-circuite le cérémonial instauré par Forteserre (un demiginguet d'amende pour tout convive qui prononcerait le nom de " majesté ") et contribue à son éducation morale : elle le corrige de sa colère grâce à un vrai-faux gobelet magique et le guérit de ses préjugés aristocratiques (sur le thème "nous sommes tous des fils de Noé »). Forteserre accepte alors de donner en mariage sa fille, Gracilie, à l'Éveillé.

- La construction du Palais Royal (appelé «Bititibi ») : les travaux d'embellissement de la petite maison de Bibi sur la frontière (à trois jours de la Capitale) prennent de l'ampleur : Titi demande d'acheter toutes les terres alentour pour bâtir à la place un magnifique Palais, mais un propriétaire terrien résiste et demande un prix exorbitant. Après de longues tractations et considérations sur le rapport entre justice, économie et politique, Titi qui ne veut ni forcer le propriétaire ni flouer les autres propriétaires en payant plus cher une parcelle qu'une autre, trouve une issue : il laisse la parcelle intacte et l'entoure d'une muraille: "Ainsi je n'ai pas tort de vous dire que ce sera le plus bel endroit du parc, puisque ce sera une marque de modération des rois, et de leur attention à ne pas violer ce qu'on appelle la propriété de leurs sujets. »

- Guerre contre Triptillon : Titi obtient la libération de la province soulevée par Tripalle et Triptillon, et élabore, avec Forteserre les fondements d'une politique commune. Le récit s'achève sur l'arrivée de Bibi dans la Capitale.

- Livre 8 (apocryphe) " qui donne une conclusion nécessaire" : on célèbre alors le triple mariage de Forteserre avec Blanchebrune (cousine de Titi), de Titi avec Bibi, et de l'Éveillé (devenu le duc de Félicie) avec la princesse Gracilie. 